\title{
Virtual Thorough QT (TQT) Trial-Extrapolation of In Vitro Cardiac Safety Data to In Vivo Situation Using Multi-Scale Physiologically Based Ventricular Cell-wall Model Exemplified with Tolterodine and Fesoterodine
}

\author{
Nikunjkumar Patel, ${ }^{1,2,3}$ Barbara Wisniowska, ${ }^{2}$ and Sebastian Polak ${ }^{1,2}$
}

Received 15 April 2018; accepted 20 June 2018; published online 11 July 2018

\begin{abstract}
QT interval prolongation typically assessed with dedicated clinical trials called thorough QT/QTc (TQT) studies is used as surrogate to identify the proarrhythmic risk of drugs albeit with criticism in terms of cost-effectiveness in establishing the actual risk of torsade de pointes (TdP). Quantitative systems toxicology and safety (QSTS) models have potential to quantitatively translate the in vitro cardiac safety data to clinical level including simulation of TQT trials. Virtual TQT simulations have been exemplified with use of two related drugs tolterodine and fesoterodine. The impact of bio-relevant concentration in plasma versus estimated heart tissue exposure on predictions was also assessed. Tolterodine and its therapeutically equipotent metabolite formed via CYP2D6 pathway, 5-HMT, inhibit multiple cardiac ion currents $\left(I_{\mathrm{Kr}}, I_{\mathrm{Na}}, I_{\mathrm{CaL}}\right)$. The QSTS model was able to accurately simulate the QT prolongation at therapeutic and supra-therapeutic dose levels of tolterodine well within $95 \%$ confidence interval limits of observed data. The model was able to predict the QT prolongation difference between CYP2D6 extensive and poor metaboliser subject groups at both dose levels thus confirming the ability of the model to account for electrophysiologically active metabolite. The QSTS model was able to simulate the negligible QT prolongation observed with fesoterodine establishing that the 5-HMT does not prolong QT interval even though it is a blocker of hERG channel. With examples of TOL and FESO, we demonstrated the utility of the QSTS approaches to simulate virtual TQT trials, which in turn could complement and reduce the clinical studies or help optimise clinical trial designs.
\end{abstract}

KEY WORDS: arrhythmia; cardiac safety; Cardiac Safety Simulator; fesoterodine; hERG; thorough QT trial; tolterodine; torsade de pointes.

\section{INTRODUCTION}

Adverse effect of drugs on cardiac electrophysiology especially incidents of torsade de pointes (TdP) is one of the most prominent cause of withdrawal of several marketed drugs towards the end of the twentieth century and beginning of the 21st (1-4). Occurrence of TdP is typically a rare adverse event; however, the consequences could be life-threatening hence there is more regulatory concern for safe use of drugs. The risk of TdP has been ascribed for drugs spanning wider therapeutic areas, other than cardiovascular drugs (5); hence, it has become important to assess the TdP risk for any new medicine. As TdP is a rare and

\footnotetext{
${ }^{1}$ Simcyp Division, Certara UK Limited, Level 2-Acero, 1 Concourse Way, Sheffield, S1 2BJ, UK.

${ }^{2}$ Unit of Pharmacoepidemiology and Pharmacoeconomics, Faculty of Pharmacy, Jagiellonian University Medical College, Krakow, Poland.

${ }^{3}$ To whom correspondence should be addressed. (e-mail: Nikunjkumar.Patel@certara.com)
}

multi-factorial event, it is very difficult to predict. A drug that is well tolerated in most patients can cause TdP in a particular individual under certain clinical situations, e.g. disease or co-medications or electrolyte imbalance (6). Thus, actual incidence of TdP can be a result of multiple clinical risk factors other than drug's ability to perturb cardiac electrophysiology making it rare and challenging to predict its risk precisely. However, as TdP is a type of polymorphic ventricular tachycardia or arrhythmia, there is an association between prolongation of QT interval of electrocardiogram (ECG) and TdP. A drug that can prolong QT interval could potentially cause TdP. Hence, QT interval prolongation by the drug is considered an acceptable biomarker of TdP risk albeit with some criticism (7). In 2006, the ICH issued a first formal guidance (E14) in this regard-"The Clinical Evaluation of QT/QTc Interval Prolongation and Proarrhythmic Potential for NonAntiarrhythmic Drugs" (8). QTc is the QT interval corrected for the heart rate variations by suitable formulae. The major aspect of the guidance was the thorough QT/QTc (TQT) study that is a dedicated and well-controlled clinical 
trial to quantify the propensity of drug to prolong QT/QTc at therapeutic and supra-therapeutic dose levels as compared to positive control. The cause of concern in TQT trial is the QT prolongation exceeding $5 \mathrm{~ms}$ determined by whether the upper boundary of the $95 \%$ confidence interval around the mean effect on the QT interval is larger than $10 \mathrm{~ms}$. QT interval varies significantly larger than $5 \mathrm{~ms}$ naturally during the day, due to meal effect, gender and hormonal effects and other factors hence to detect such a small difference in a parameter (QT interval) with inherently high basal variability, the study is typically well controlled for baseline, placebo and time of day (circadian) effects and includes positive control (typically moxifloxacin). QT varies with heart rate hence various correction formulae are applied to correct QT for heart rate changes (known as QTc) among which the Fridericia method is widely used (9). E14 guidelines are adopted by many regulatory agencies and TQT trials have become routine in new drug approval process.

There are no drugs withdrawn from the market due to TdP risk after introduction of TQT trials; however, there are concerns that the optimal fail fast fail safe paradigm in recent drug development programmes lead to unnecessary removal of therapeutically promising molecules at early stage of development due to perceived safety risk (10). There are also doubts regarding the cost-effectiveness of the TQT trials in identifying the TdP risk; therefore, alternative approaches are being evaluated (11). Moreover, the number of reports of TdP remains significant indicating there is a translational gap in estimating the real-world TdP risk of new drugs (4). Thus, translational tools that allow prediction of clinical cardiac risk and clinical QT response are greatly needed $(6,12)$. One such set of tools are physiologically based, biophysically detailed models of cardiac physiology that can characterise the contributions of multiple ion channel inhibition on the electrophysiology of human cardiomyocytes incorporated in the physical model of ventricular wall as string of cells arranged from inside to outside of ventricular wall. These multi-scale quantitative systems toxicology and safety (QSTS) models can bridge the translational gap in cardiac safety assessment with the use of in vitro dynamic ion channel inhibition data and expected exposure levels to simulate subsequent effects on ECG profile in virtual patients, which represent an array of physiologies expected in clinical practice $(13,14)$. Such QSTS approach allows mechanistic in vitro to in vivo extrapolation (IVIVE) of preclinical cardiac safety assessment to clinical cardiac safety risk and can aid in bridging the translational gap in TdP risk assessment (15). Recently, we have used the QSTS models to predict quantitatively the QT prolongation effect of a drug influencing multiple cardiac ion channels with electrophysiologically active metabolite(s) such as citalopram (12) and moxifloxacin, drug widely used as positive control in TQT trials (6). More of such examples will allow for more confidence and pave a path towards establishing mechanistic simulations in bridging the existing translational gap in preclinical to clinical cardiotoxicity. Here, we report utilisation of such QSTS platform, the Cardiac Safety Simulator (CSS) (14,16), that combines a mechanistic electrophysiological model of human left ventricular cardiomyocytes with the database of human physiological, genotypic and demographic data for the prediction of human QT prolongation, for tolterodine (TOL) and its therapeutically equipotent metabolite 5hydroxymethyl tolterodine (5-HMT). Both TOL and 5HMT are known to interact with multiple cardiac ion channel currents (http://www.ema.europa.eu/docs/en_GB/ document_library/EPAR_-_Scientific_Discussion/human/ 000723/WC500040181.pdf). As the CSS platform also allows to propagate population variability in pharmacokinetics (drug exposure) towards pharmacodynamics (cardiac activity), we also simulated a virtual TQT study for therapeutic (2 $\mathrm{mg})$ and supra-therapeutic (4 mg) dose of tolterodine along with moxifloxacin (MOXI) $400 \mathrm{mg}$ given orally as a positive control and compared the simulation results with reported clinical TQT study results (17). In a separate virtual TQT study (18), the cardiac effect of fesoterodine (FESO), an ester pro-drug of 5-HMT (19), was simulated. Therapeutically recommended (4 $\mathrm{mg})$ and supra-therapeutic $(28 \mathrm{mg})$ doses of FESO were combined with $400 \mathrm{mg}$ MOXI used as positive control in this clinical TQT trial.

\section{MATERIALS AND METHODS}

\section{Pharmacology of the Tested Drugs}

5-HMT is formed by metabolism of TOL via cytochrome P450 (CYP) 2D6 enzyme $(17,20)$, which is known to have genotypic and phenotypic differences in human populations (21). 5-HMT itself is metabolised by predominantly via CYP2D6 and CYP3A4 pathways with minor contribution of renal excretion. The clinical TQT study (17) identified the extensive metaboliser (EM) and poor metaboliser (PM) phenotype status of subjects and reported the exposure levels for both groups and the subsequent QT prolongation. Natural frequency of PM phenotype in Caucasian population is around $8 \%$ (Simcyp in-house meta-analysis data). However, the clinical study recruited around $45 \%$ subjects with PM status to include sufficient representation of PM group in the studied cohort. We also simulated EM and PM groups with similar number of subjects as clinical study and verified the predictions with clinical data. The influence of exposure in systemic circulation versus estimated exposure in heart tissue was also studied in the current work.

Fesoterodine is an ester pro-drug of 5-HMT. FESO was designed to produce therapeutically active metabolite (5-HMT) of TOL avoiding the CYP2D6 polymorphism-related variability in pharmacokinetics in exposure levels in patients because the conversion of FESO to 5-HMT is driven by ubiquitous esterase enzymes (19). FESO converts rapidly and almost completely to 5-HMT in systemic circulation as evident from the observation that the FESO is almost undetectable in systemic circulation after oral administration (18).

\section{Clinical Pharmacokinetic and QT Prolongation Data}

\section{Tolterodine}

Bio-relevant exposure levels of active chemical moieties along with ion channel activity are required as input to the CSS 
system to predict the QT interval prolongation effect. Malhotra et al. (17) have reported results from a thorough QT trial at therapeutically recommended ( $2 \mathrm{mg}$ BID) and supra-therapeutic (4 mg BID) dose of TOL with $400 \mathrm{mg}$ MOXI as positive control. The steady-state (day 4) maximum plasma concentration $\left(C_{\text {max,Ss }}\right)$ for TOL and metabolite 5-HMT after 2- and 4-mg BID dose levels for CYP2D6 EM and PM subject groups along with $C_{\text {max,Ss }}$ for MOXI treatment were obtained from Malhotra et al. (17). Virtual subjects were generated based on the demographic information (age, gender and body weight) provided in the clinical study based on bootstrapping method. The population variability in drug exposure parameters (here, $C_{\text {max,SS,TOL }}$ and $C_{\text {max,SS,5-HMT }}$ for both EM and PM groups) was simulated using the bootstrapping method based on the mean and variance of population pharmacokinetics (PK) parameters reported in the study. The population variability in PK was then propagated into the CCS platform to predict the QT prolongation for the virtual trial population. The placebo effect was simulated as the QT prolongation response with zero drug concentration for a given subject at given time point. Heart tissue to plasma partition coefficient ( $\left.K_{\text {heart:plasma }}\right)$ was estimated with 'Method 3' in Simcyp Simulator V16 (Certara UK Limited, Sheffield, UK) where the 'bottom-up' predicted volume of distribution at steady state $\left(V_{\mathrm{ss}}\right)$ from physicochemical parameters of the chemical moieties was calibrated with observed $V_{\text {ss }}$ for both TOL and 5MHT using 'Kp scalar'. 'Kp scalar' is a multiplier to the estimated tissue:plasma partition coefficients from 'Method 3' of Simcyp Simulator. The observed $V_{\mathrm{ss}}$ for TOL was $1.66 \mathrm{~L}$ per kg of body weight (22) while the predicted $V_{\text {ss }}$ from 'Method 3' from physicochemical parameters was $2.8 \mathrm{~L} / \mathrm{kg}$. With 'Kp scalar' value of 0.75 , the predicted $V_{\text {ss }}$ matched well to the observed $V_{\text {ss. }}$. With adjusted 'Kp scalar' to match the observed $V_{\mathrm{ss}}$, the estimated $K_{\text {heart:plasma }}$ for TOL was 0.48 and the fraction unbound in the heart tissue $\left(f_{\mathrm{u}, \mathrm{Heart}}\right)$ from 'Method 3' was 0.066 . Similarly, the 'Kp scalar' for 'Method 3' was adjusted to 0.46 to match the observed $V_{\text {ss }}$ of $1.73 \mathrm{~L} / \mathrm{kg}$ for 5 -HMT (23). With adjusted 'Kp scalar' to match the observed $V_{\text {ss }}$, the estimated $K_{\text {heart:plasma }}$ for 5-HMT was 2.93 and the $f_{\mathrm{u}, \text { Heart }}$ from 'Method 3' was 0.088 . These estimated values of $K_{\text {heart:plasma }}$ and the $f_{\mathrm{u} \text {,Heart }}$ for TOL and 5-HMT were used to calculate the total and unbound heart tissue concentration of both moieties from the observed plasma concentration data.

\section{Moxifloxacin}

Daily dose of $400 \mathrm{mg}$ MOXI was administered orally to volunteers in positive control arm of the TQT trial and QT prolongation was estimated on day 4. We have previously developed a PBPK-QSTS model for MOXI (6). We used the same PBPK model of MOXI and ran simulation for multiple daily doses of $400 \mathrm{mg}$ and calculated the maximum unbound heart tissue exposure levels on day 4 and input to the CSS platform to drive the QT response. The maximum QTc prolongation was calculated from simulated study arm and compared with the reported QTcF prolongation in the TQT study report.

\section{Fesoterodine}

Malhotra et al. (18) have reported results from a thorough QT trial of FESO at therapeutically recommended (4 mg sustained release tablet once a day for three days) and supra-therapeutic (28 mg, seven sustained release tablets of $4 \mathrm{mg}$ FESO given once a day for three days) dose of FESO with $400 \mathrm{mg}$ MOXI as positive control. The steady-state (day 3) maximum plasma concentration $\left(C_{\max , \mathrm{Ss}}\right)$ for active metabolite 5-HMT after 4and 28-mg dose levels were obtained from Malhotra et al. (18). Similar to TOL, virtual subjects population variability in drug exposure parameters (here, $C_{\max , \mathrm{Ss}, 5-\mathrm{HMT}}$ ) were generated based on bootstrapping method. The population variability in PK was then propagated into the CCS platform to predict the QT prolongation for the virtual trial population. The placebo effect was simulated as the QT prolongation response with zero drug concentration for a given subject at given time point. Simulations with only unbound plasma concentration as operating concentration were studied for FESO.

\section{In Vitro/In Silico Cardiac Safety Data}

TOL is known to inhibit at least three cardiac ion currents namely $I_{\mathrm{Kr}}$ (rapid delayed rectifying current), $I_{\mathrm{Na}}$ and $I_{\mathrm{CaL}}$ (sodium and late calcium currents respectively) that are important in cardiac electrophysiology (http:// www.ema.europa.eu/docs/en_GB/document_library/ EPAR_-_Scientific_Discussion/human/000723/ WC500040181.pdf). Its metabolite, 5-HMT, is also known to inhibit at least the same three cardiac ion channel currents as the parent albeit with different potency (http:// www.ema.europa.eu/docs/en_GB/document_library/ EPAR_-_Scientific_Discussion/human/000723/ WC500040181.pdf). Concentration of the ligands required to inhibit $50 \%$ of the current of studied ion channel $\left(I C_{50}\right.$ values) of TOL and 5-HMT for $I_{\mathrm{Kr}}, I_{\mathrm{Na}}$ and $I_{\mathrm{CaL}}$ currents are reported in Table I.

\section{Simulation Methodology}

CSS version 2.1 (Certara UK Limited, Sheffield, UK) was used to perform simulations. The ten Tusscher 2006 model (24) of cardiomyocte electrophysiology implemented in CSS platform was used to model drug effect on ion channels at cell level. The CSS platform considers the heterogeneities in ionic currents between endocardial, midmyocardial and epicardial cells by one-dimensional fibre (ventricular wall thickness) paced at the epicardial side. The 50:30:20 distribution of the endo-, mid- and epicardial cells was used to compose the ventricular wall thickness together with the diffusion coefficient equal to $0.0016 \mathrm{~cm}^{2} / \mathrm{ms}$. The forward Euler method was used to integrate model equations. First and last beats were excluded from the simulated pseudoECG to provide stable signal. An ECG trace produced by the model is termed pseudoECG as it misses the $\mathrm{P}$ wave because the model only accounts for the ventricular cell wall. A space step and a time step were set to $\Delta x=0.01 \mathrm{~mm}$ and $\Delta t=$ $0.01 \mathrm{~ms}$, and total simulation time was set to $10,000 \mathrm{~ms}$ in all cases. To account for the drug-triggered ionic currents modifications, the specific equation describing current of interest was multiplied by the inhibition factor accordingly to the in vitro values provided by the literature search describing the concentration-dependent ionic current 
Table I. Cardiac ion channel inhibition data used in simulations and its source

\begin{tabular}{|c|c|c|c|c|}
\hline \multirow[t]{2}{*}{ Moiety } & \multicolumn{4}{|c|}{$I C_{50}$ in $\mu \mathrm{M}$ (Hill equation coefficient $\left.{ }^{\$}\right)$} \\
\hline & $I_{\mathrm{Kr}}$ & $I_{\mathrm{Na}}$ & $I_{\mathrm{CaL}}$ & Reference \\
\hline TOL & $0.011(1)$ & $4.85(1)$ & $560(1)^{*}$ & $\begin{array}{l}\text { (http://www.ema.europa.eu/docs/en_GB/document_library/EPAR_-_Scientific_Discussion/human/ } \\
\text { 000723/WC500040181.pdf) }\end{array}$ \\
\hline $\begin{array}{l}5- \\
\text { HMT }\end{array}$ & $0.39(1)$ & $17.25(1)$ & $560(1)^{\wedge}$ & $\begin{array}{l}\text { (http://www.ema.europa.eu/docs/en_GB/document_library/EPAR_-_Scientific_Discussion/human/ } \\
\text { 000723/WC500040181.pdf) }\end{array}$ \\
\hline
\end{tabular}

*Calculated $I C_{50}$ within the CSS platform based on \%inhibition versus concentration profile reported in reference $(17 \%$ inhibition at $15 \mu \mathrm{M}$ concentration of TOL); $\wedge$ calculated $I C_{50}$ within the CSS platform based on \%inhibition versus concentration profile reported in reference (18\% inhibition at $15 \mu \mathrm{M}$ concentration of TOL); ${ }^{\$}$ Hill coefficient was not reported in the study hence assumed to be 1 in all cases

inhibition. The inhibition factor was calculated with the use of the Hill equation [Eq. 1].

Inhibition factor $=\frac{1}{1+\left(I C_{50} / \text { drug concentration }\right)^{n}}$

where $I C_{50}$ is the concentration responsible for the $50 \%$ inhibition of the ionic current, $n$ is the Hill equation parameter, drug concentration is the active drug concentration $[\mu \mathrm{M}]$.

The population variability of other physiological parameters was mimicked by applying the virtual population generator as described previously $(25,26)$. The circadian heart rate variability model has recently been incorporated into the CSS system to enable assessment of circadian variations in heart rate on the estimate of QT prolongation (27). To account for the circadian plasma ions, a variability model describing the gender-dependent diurnal fluctuation of the three main ions $\left(\mathrm{Ca}^{2+}, \mathrm{K}^{+}\right.$and $\left.\mathrm{Na}^{+}\right)$has been applied $(28,29)$. CSS allows simulation of clinical scenarios inclusive of effect of circadian variation on predicted QT which can aid in understanding potential between subjects and within subject variations in QT response prior to costly and time-intensive TQT clinical studies.

Placebo and heart rate-corrected QT interval prolongation $(\Delta \Delta \mathrm{QTc})$ was calculated from simulated data and compared with reported values from clinical TQT trials. The CSS platform already outputs the heart rate-corrected QT interval by Fridericia formulae (QTcF). The simulations included placebo (null) simulation, where additional simulation was run with zero drug concentration for each individual and at each simulated time point. $\Delta \Delta \mathrm{QTcF}$ was calculated for simulation results by subtracting the QTcF in absence of drug from the value of QTcF in presence of drug.

\section{RESULTS}

\section{Tolterodine}

The clinically observed $\Delta \Delta \mathrm{QTcF}$ (ms) at 2- and 4-mg BID dose levels for population of 48 subjects was reported in study by Malhotra et al. (17). Sweeney et al. (30) have analysed the TQT trial clinical data using population PKPD model to estimate the population mean of EM and PM groups. The simulated QT prolongation data of 48 subjects, separated by EM $(N=27)$ and $\mathrm{PM}(N=21)$ groups, are reported along with clinical $\Delta \Delta \mathrm{QTcF}$ data for 2- and 4-mg dose groups in Fig. 1a, b, respectively. The simulations were run using four concentration levels to study the most biorelevant exposure surrogate to explain the observed $\Delta \Delta \mathrm{QTcF}$ data-(i) total plasma concentration; (ii) unbound plasma concentration; (iii) total heart tissue concentration and (iv) unbound heart tissue concentration. The results clearly showed that the total heart tissue and total plasma concentrations over-estimated the $\Delta \Delta \mathrm{QTcF}$. There was no significant difference between the predictions using the unbound plasma and unbound heart tissue concentrations mainly because the estimated partition coefficient for TOL between heart tissue and plasma is approximately 0.45 and the $\mathrm{fu}_{\mathrm{Heart}}$ of 0.066; thus, unbound heart tissue and plasma exposures were similar. The predictions using the unbound plasma and unbound heart tissue exposure levels were closest to clinically observed $\Delta \Delta \mathrm{QTcF}$ for both EM and PM groups and at both dose levels (Fig. 1).

Figure 2 shows the comparative $\Delta \Delta \mathrm{QTcF}$ prolongation from simulated and clinical TQT trial results for therapeutic and supra-therapeutic dose levels of TOL along with positive control MOXI. The vertical error bar indicates the $95 \%$ confidence interval around the mean $\Delta \Delta \mathrm{QTcF}$ from the clinical study and simulated population.

The concentration- $\Delta \Delta \mathrm{QTcF}$ relationships for both dose levels and by EM and PM groups are provided in Fig. 3. Figure $3 \mathrm{a}$, b shows the relationship for 2- and 4-mg BID dose levels while Fig. 3c, d shows the relationship for EM and PM groups, respectively. There is no considerable difference in slopes of concentration- $\Delta \Delta \mathrm{QTcF}$ relationship, which ranged from 10 to $15 \mathrm{~ms} / \mathrm{ng} / \mathrm{mL}$.

Figure 3 clearly shows poor relationship between 5-HMT concentration and $\Delta \Delta \mathrm{QTcF}$ indicating that the metabolite 5HMT had negligible contribution to the predicted QT prolongation while the TOL concentration was linearly and positively correlated with the $\Delta \Delta \mathrm{QTCF}$. EM subjects showed larger values of slope than the PM subjects (approx. $5 \mathrm{~ms} / \mathrm{ng}$ / $\mathrm{mL}$ larger) while the lower dose had slightly higher slope than the higher dose level.

\section{Fesoterodine}

The clinically observed $\Delta \Delta \mathrm{QTcF}$ (ms) at 4- and 28-mg FESO dose levels were reported in study by Malhotra et al. (18). Figure 4 shows comparative values of $\Delta \Delta \mathrm{QTcF}$ (ms) 


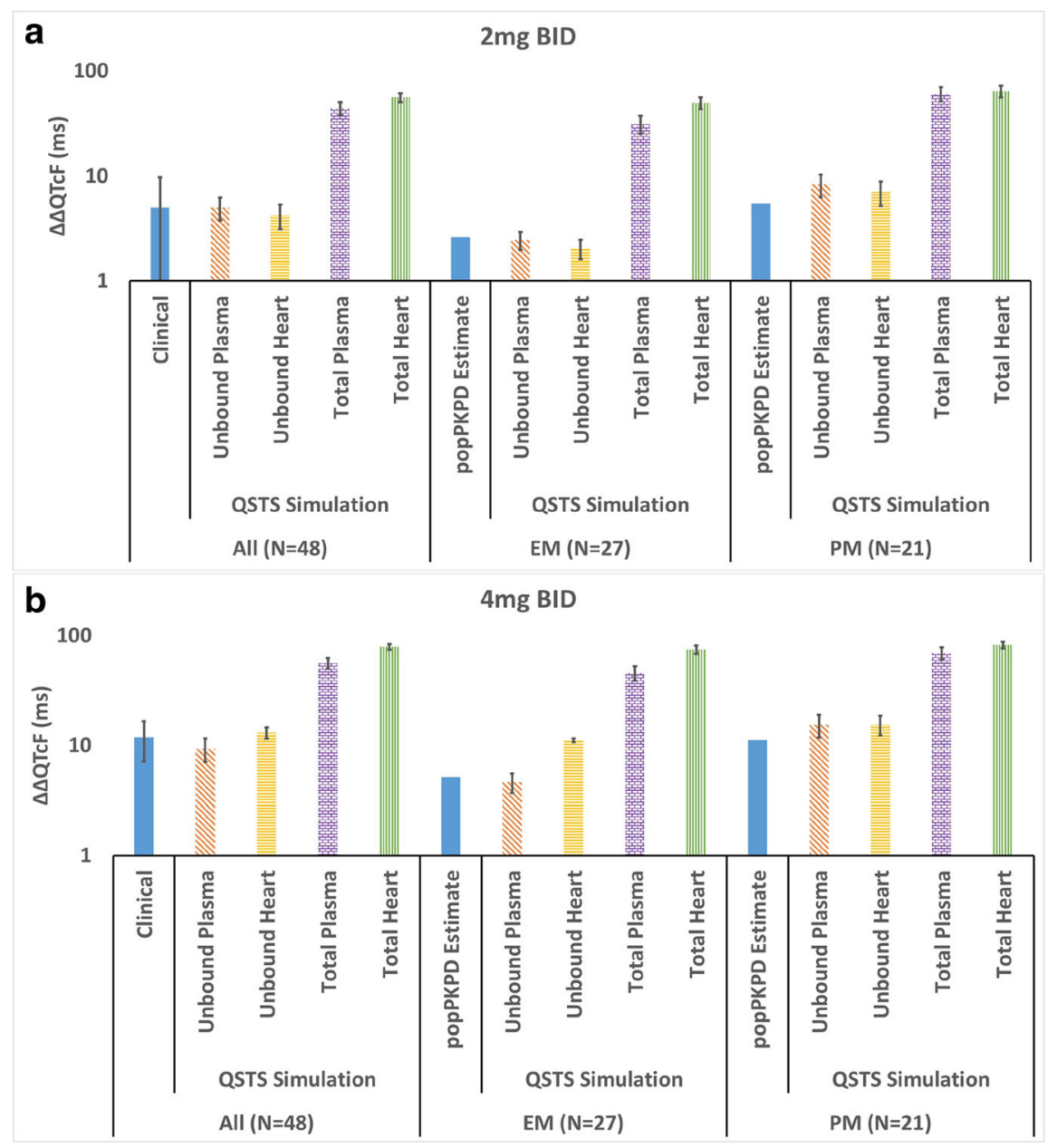

Fig. 1. Observed and simulated $\Delta \Delta \mathrm{QTcF}$ (ms) for a 2-mg BID and b 4-mg BID dose levels. Blue solid bars represent observed data, orange diagonal striped bars represent the simulated data with unbound plasma concentration as operating concentration, yellow horizontal striped bars represent simulated data with unbound heart tissue concentration as operating concentration, purple brick patterned bars represent the simulated data with total plasma concentration as operating concentration, green vertical striped bars represent simulated data with total heart tissue concentration as operating concentration. Observed data for extensive and poor metabolizer groups was obtained by population pharmacokinetic-pharmacodynamic (popPKPD) modelling of clinical data. The black thin error bars represent the $95 \%$ confidence interval around the mean value

from simulated trial and observed data on day 3 at both dose levels.

The model predicted the QT interval prolongation really well at lower dose level of $4 \mathrm{mg}$; however, the clinical results showed no change or slight shortening of QTcF interval ($0.1 \mathrm{~ms}$ ) at higher dose of $28 \mathrm{mg}$ while the model predicted small QT prolongation $(3 \mathrm{~ms}$ ). The simulated variability was much smaller than that observed in the clinical trial and the simulated results are within the $95 \%$ confidence interval reported in the clinical study (also showed as vertical error bars in Fig. 4). Thus, the results based only on in vitro cardiac safety input data is in good agreement with clinical TQT trial.

\section{DISCUSSION}

TOL, an antimuscarinic agent used for the treatment of overactive bladder, has an equipotent metabolite 5-HMT. TOL is found to be a potent inhibitor of hERG channel while the metabolite 5-HMT is a magnitude less potent in inhibiting hERG. Both TOL and 5-HMT inhibit late calcium channel although with very low potency. The unbound fraction of TOL and 5-HMT in plasma is around 3.7 and $36 \%$, respectively (17). Therefore, even though the exposure levels of TOL are many-fold higher in CYP2D6 PM subjects, the effective free plasma exposure of net therapeutically active moieties are comparable in EM and PM groups negating the need for dose adjustment for PM group. However, the cardiac safety profile could be different due to significant difference in potency of TOL and 5-HMT in inhibiting the hERG channel. As the TOL is $>10$-fold more potent hERG inhibitor than 5-HMT, a considerably higher QT prolongation is expected in PM subject group. However, simulated results as well as clinical observations indicate only a small increase in QT prolongation in PM subjects as compared to EM subjects mainly due to much higher binding of TOL to plasma proteins moderating the increase in total plasma 


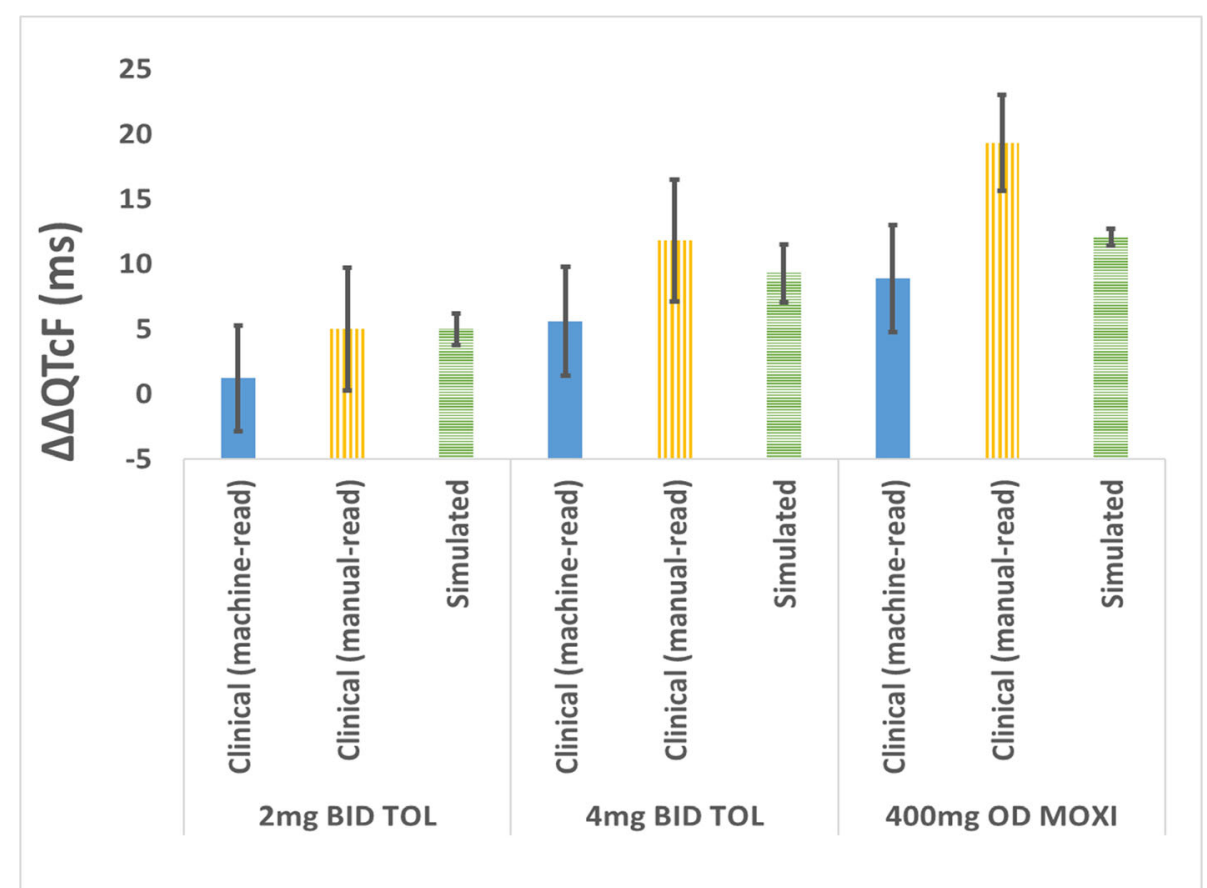

Fig. 2. Clinically observed and simulated $\Delta \Delta \mathrm{QTcF}$ (ms) at therapeutic and supra-therapeutic dose levels of tolterodine along with positive control drug moxifloxacin for thorough QT trial of tolterodine. Clinical $\Delta \Delta \mathrm{QTcF}$ calculated from machine-read electrocardiogram is represented with solid blue bar, clinical $\Delta \Delta \mathrm{QTCF}$ calculated from manual-read electrocardiogram is represented with vertically striped yellow bar, and simulated $\Delta \Delta \mathrm{QTcF}$ is represented with horizontally striped green bar. The black thin error bars represent the $95 \%$ confidence interval around the mean value
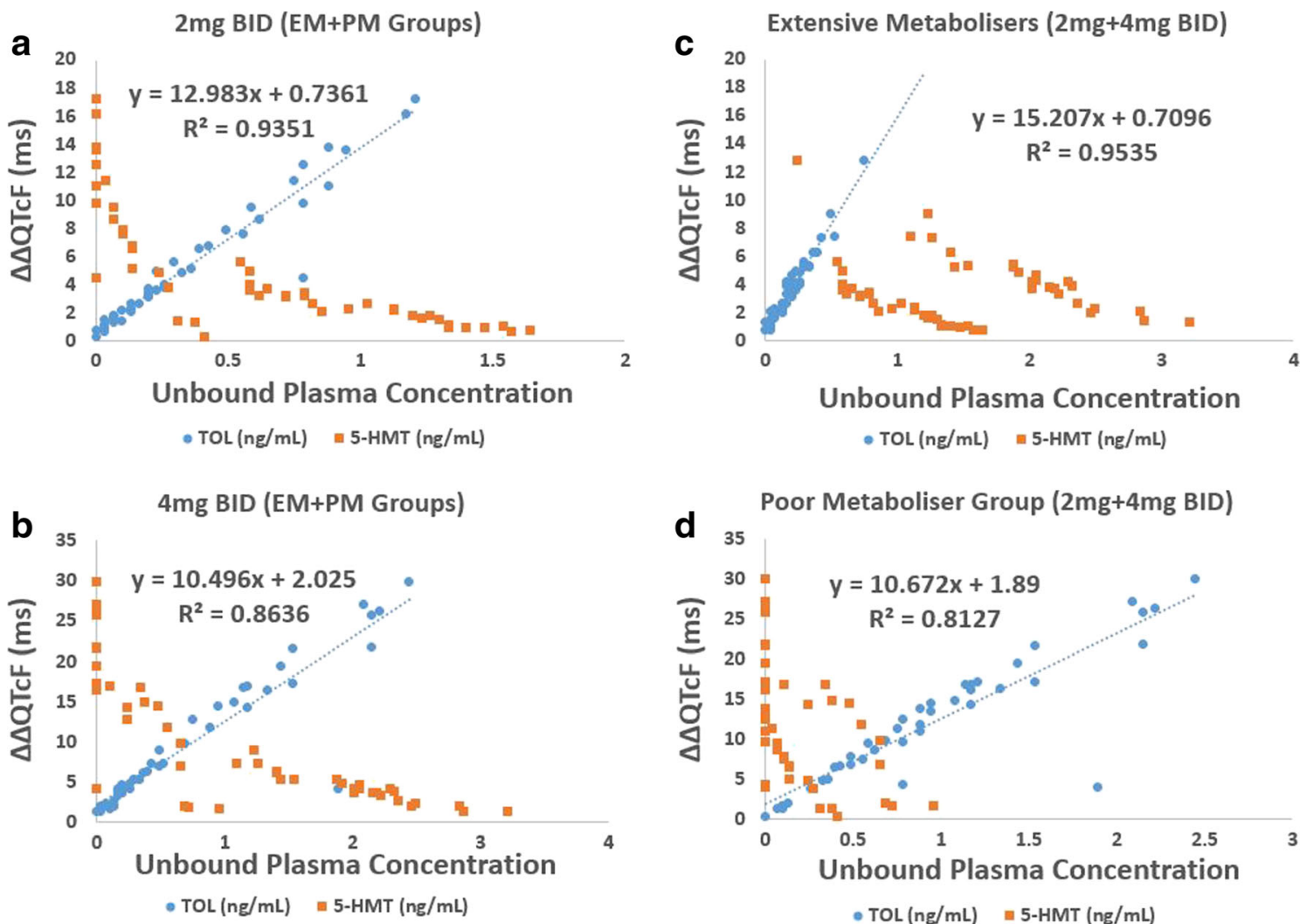

Fig. 3. The concentration- $\Delta \Delta \mathrm{QTcF}$ relationships for tolterodine and metabolite 5-hydroxymethytolterodine after administration of 2-mg BID dose (a) and 4-mg BID dose (b) of tolterodine and for extensive metabolizer population group (c) and poor metabolizer population groups (d) 


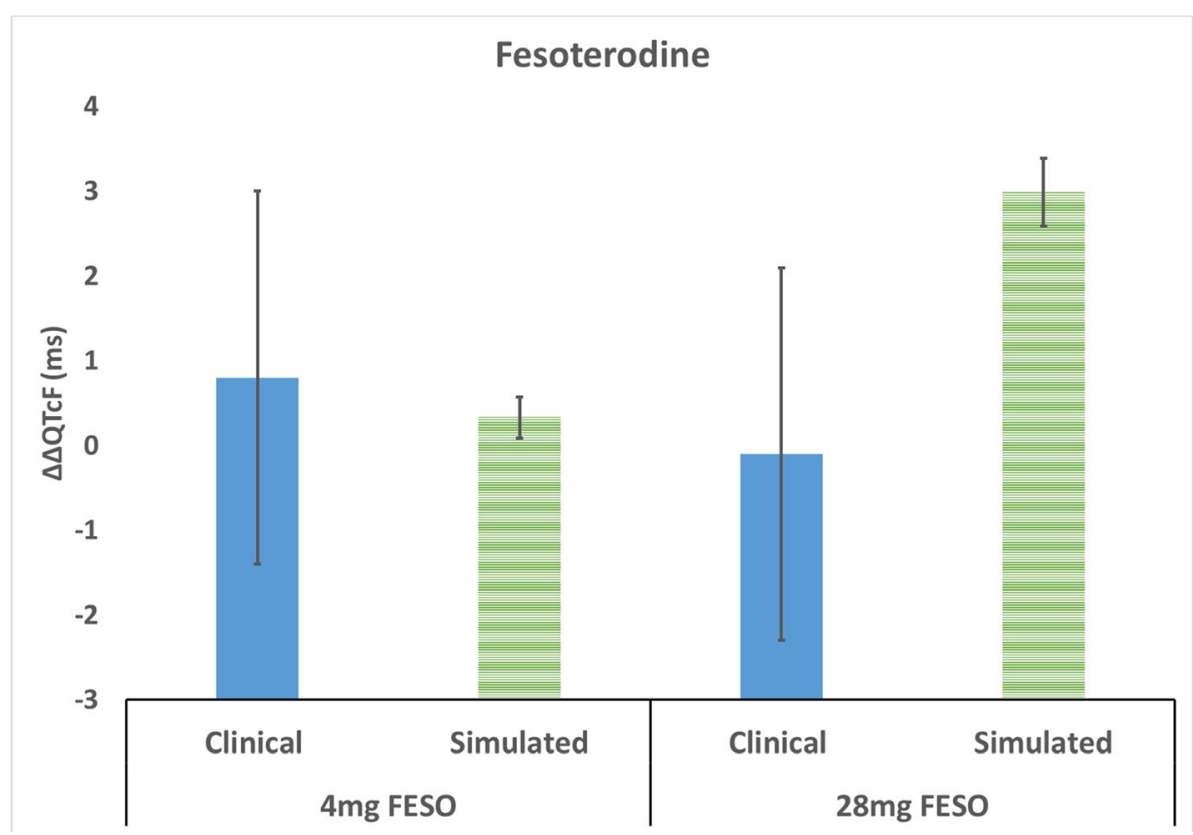

Fig. 4. Clinically observed and simulated $\Delta \Delta \mathrm{QTcF}(\mathrm{ms})$ at therapeutic $(4 \mathrm{mg}$ ) and supratherapeutic dose $(28 \mathrm{mg}$ ) levels of fesoterodine in thorough QT trial. Clinically observed $\Delta \Delta \mathrm{QTcF}$ is represented with solid blue bar and simulated $\Delta \Delta \mathrm{QTcF}$ is represented with horizontally striped green bar. The black thin error bars represent the $95 \%$ confidence interval around the mean value

concentration in PM subjects. Natural frequency of CYP2D6 $\mathrm{PM}$ polymorphism in normal population is around $8 \%$; however, the clinical study, and hence the simulated trial, was enriched with higher proportion of PM (45\%) subjects to sufficiently represent the high-risk PM group subjects. The simulation results indicated that the QSTS model accurately predicted the clinical QT prolongation not only for therapeutic and supra-therapeutic dose levels but also was able to differentiate the effect of EM and PM exposure levels on QT prolongation. It is also important to note that the clinically observed QT interval prolongation estimated with machineread ECG is systematically lower than the manual-read ECG (Fig. 2). When the QSTS model is assessed in terms of its predictive performance for such smaller values of QT interval prolongation, it becomes important to understand the variability and uncertainty associated with the clinical data and/or the measurement technique. There were considerable differences in QT prolongation from clinical study noted between manually read and machine ECG; hence, both machine-read and manual-read values were provided along with predicted results (Fig. 2). For MOXI arm, the simulated results were within the reported $90 \%$ confidence interval of clinical data from machine-read ECG while higher than the reported value with manually read ECG (17). We have previously shown that there exists inter-study variability in QT interval prolongation after $400 \mathrm{mg}$ MOXI once-daily (OD) treatment due to multiple of study design factors such as encapsulation of tablet for purpose of blinding and manufacturer of the formulation, etc. (6). We have shown there that the prediction for MOXI was reasonably good at much wider pooled clinical data set from six independent studies; thus, here, we consider the prediction for MOXI positive arm acceptable. For TOL $2 \mathrm{mg}$ BID as well as $4 \mathrm{mg}$ BID, the simulated results were well within $95 \%$ confidence interval of clinically reported values from both machine-read and manual-read ECG results. There are no animal or human studies reporting the heart tissue exposure of TOL or 5-HMT; hence, the simulated heart tissue exposure cannot be fully verified. We already discussed at length the issues and uncertainties around the bio-relevant exposure levels driving the cardiac response and limited knowledge in public literature. We previously studied the impact of model selection on MOXI, for example, the O'Hara Rudy 2011 model (31) tends to predict larger QT prolongation than the ten Tusscher 2006 model (24) for the same input parameters (6); however, well-defined systematic studies are needed to analyse such model comparison at single cell level (action potential duration) as well as ventricular wall level (QT prolongation) simulations to provide further recommendations. More experimental data at both in vitro and in vivo level and collaborative research initiatives are required to complement QSTS modelling results to test or establish hypotheses and move the field of cardiac risk assessment forward.

Another important measure to assess the predictive performance of the QSTS model would be to compare its ability to simulate the effect $(\Delta \Delta \mathrm{QTcF})$ versus exposure (drug concentration) profile. The slope of simulated $\Delta \Delta \mathrm{QTcF}$-concentration relationship (10-15 ms/ng/mL) was very similar to that reported from clinical data analysis (population mean point estimate of $13.4 \mathrm{~ms} / \mathrm{ng} / \mathrm{mL}$ ) for unbound plasma concentration. Moreover, the QSTS model showed no considerable difference in EM and PM groups similar to the observation made from clinical data analysis by Sweeney et al. (30). The contribution of metabolite 5-HMT in QT prolongation was negligible as evident from no correlation between 5-HMT concentration and QT prolongation at both dose levels and for both EM and PM groups. This observation from the simulated results can also be verified with another TQT study report for FESO, the ester pro-drug of 5-HMT. Clinical TQT trial of FESO with 4-mg 
and 28-mg dose resulted in negligible QT prolongation or slightly shorter QT prolongation as compared to placebo. The simulations predicted similarly low to negligible QT prolongation at both dose levels of FESO. This clearly establishes the outcome of simulation results from TOL simulation that the 5-HMT does not prolong QT interval even though it inhibits hERG channel. Ability to inhibit the calcium channel could explain the slight shortening of the QT interval in clinical TQT trial especially at higher dose level (28 mg FESO) because the potency of 5-HMT to inhibit the calcium channel is very low (Table I); thus, measurable inhibition of calcium current can only be achieved at higher exposure levels. Simulations, although recovered the observed QT prolongation range of both dose levels really well, could not mimic the QT shortening trend with dose. Although not statistically significant, the clinical data showed slightly lower QT prolongation at higher dose of FESO than at lower dose. This could be due to fact that the $I C_{50}$ value for calcium channel inhibition was not available as the maximum concentrations studied in vitro lead to around $17 \%$ inhibition of calcium current thus making it difficult to derive accurate $I C_{50}$ value. More accurate $I C_{50}$ value for calcium channel inhibition may allow the QSTS model to recover the QT shortening effect at higher exposure levels. The potency of TOL and 5-HMT is similar in inhibiting the calcium current based on limited data available from literature (http://www.ema.europa.eu/docs/en_GB/ document_library/EPAR_-_Scientific_Discussion/human/ 000723/WC500040181.pdf). As observed with lower slope of $\Delta \Delta \mathrm{QTcF}$-concentration profile at higher dose $(4 \mathrm{mg})$ compared to the slope at lower dose $(2 \mathrm{mg})$ in Fig. 3, the QSTS model was able to estimate the trend of reduction in QT prolongation effect at higher dose levels based on limited input data for calcium channel inhibition. Thus, the success of the QSTS model to predict the clinical QT interval modification by drug strongly depends on quality and accuracy of input data to the IVIVE methodology.

We showed previously that the metabolites of citalopram were very important in predicting the QT prolongation profile of citalopram (12); however, here, the metabolite 5HMT did not contribute to QT prolongation after TOL treatment even though 5-HMT was a moderate hERG channel blocker. Thus, QSTS approaches also allow testing of various hypotheses such as the impact of metabolite on safety and tolerability of the drug treatment. QSTS models can be applied at early discovery levels as the models can be fed with chemical structure to predict the QT prolongation with use of quantitative structure activity relationship (QSAR) techniques. As the drug progresses in the development, the model can be enriched with better quality input data, and the virtual TQT trials can be simulated when sufficient knowledge on the PK of drug and metabolites becomes available along with in vitro cardiac safety assessment profiles, as it was presented in the current study. Once sufficiently verified, such QSTS models can also be used to refine and optimise the clinical study designs.

\section{CONCLUSIONS}

With examples of TOL and FESO, we demonstrated the utility of the QSTS approaches to bridge the translational gap in cardiac safety assessment and simulate virtual TQT trials. Such simulations could complement and reduce the clinical studies or help optimise clinical trial designs. Such QSTS approaches can also help build and test hypothesis such as assessing the role of unbound versus total plasma concentration or assessing the contribution of electrophysiologically active metabolite and allow understanding impact of population variability such as genetic polymorphism on the cardiac safety profile of drugs. Population-based QSTS models could also help address late-stage development questions such as understanding the impact of metabolic drug-drug interactions on cardiac safety risk and identify the high-risk clinical situations for potential TdP. Adoption of such QSTS modelling strategies from early stages of drug discovery and development and enrichment of model and information along with drug product life cycle could allow bridging translational gap in clinical cardiac safety assessment. More case examples of drugs with varying PK and electrophysiological profiles are needed to build confidence in such strategy for implementation in drug development and regulatory decision-making.

\section{ACKNOWLEDGEMENTS}

The Cardiac Safety Simulator and Simcyp Simulator are freely available, following completion of the relevant workshop, to approved members of academic institutions and other nonfor-profit organisations for research and teaching purposes.

\section{AUTHOR CONTRIBUTION}

Participated in research design: NP, SP

Conducted Experiments: NP, SP

Performed data analysis: NP

Contributed to the writing of the manuscript: NP, SP, BW

\section{COMPLIANCE WITH ETHICAL STANDARDS}

Conflict of Interest NP and SP are employees of Certara UK Limited, a company that provided the CSS and Simcyp Simulator software.

Open Access This article is distributed under the terms of the Creative Commons Attribution 4.0 International License (http://creativecommons.org/licenses/by/4.0/), which permits unrestricted use, distribution, and reproduction in any medium, provided you give appropriate credit to the original author(s) and the source, provide a link to the Creative Commons license, and indicate if changes were made.

\section{REFERENCES}

1. Piccini JP, Whellan DJ, Berridge BR, Finkle JK, Pettit SD, Stockbridge N, et al. Current challenges in the evaluation of cardiac safety during drug development: translational medicine meets the Critical Path Initiative. Am Heart J. 2009;158(3):317-26.

2. Redfern W, Ewart L, Hammond T, Bialecki R, Kinter L, Lindgren $\mathrm{S}$, et al. Impact and frequency of different toxicities throughout the pharmaceutical life cycle. Toxicologist. 2010;114(S1):1081.

3. Laverty HG, Benson C, Cartwright EJ, Cross MJ, Garland C, Hammond T, et al. How can we improve our understanding of 
cardiovascular safety liabilities to develop safer medicines? Br J Pharmacol. 2011;163(4):675-93. https://doi.org/10.1111/j.14765381.2011.01255.x.

4. Stockbridge N, Morganroth J, Shah RR, Garnett C. Dealing with global safety issues : was the response to QT-liability of non-cardiac drugs well coordinated? Drug Saf. 2013;36(3):16782. https://doi.org/10.1007/s40264-013-0016-z.

5. Yap YG, Camm AJ. Drug induced QT prolongation and torsades de pointes. Heart. 2003;89(11):1363-72.

6. Patel N, Hatley O, Berg A, Romero K, Wisniowska B, Hanna $\mathrm{D}$, et al. Towards bridging translational gap in cardiotoxicity prediction: an application of progressive cardiac risk assessment strategy in TdP risk assessment of moxifloxacin. AAPS J. 2018;20(3):47. https://doi.org/10.1208/s12248-018-0199-4.

7. Hondeghem LM. Drug-induced QT prolongation and torsades de pointes: an all-exclusive relationship or time for an amicable separation? Drug Saf. 2018;41(1):11-7. https://doi.org/10.1007/ s40264-017-0584-4.

8. Guideline, ICH Harmonised Tripartite Guideline E14. The clinical evaluation of QT/QTc interval prolongation and proarrhythmic potential for non-antiarrhythmic drugs E14, [2014-03]. 2005. Available at:http://www.ich.org/fileadmin/ Public_Web_Site/ICH_Products/Guidelines/Efficacy/E14/ E14_Guideline.pdf.

9. Fridericia LS. Die Systolendauer im Elektrokardiogramm bei normalen Menschen und bei Herzkranken. J Intern Med. 1920;53(1):469-86.

10. Paul SM, Mytelka DS, Dunwiddie CT, Persinger CC, Munos $\mathrm{BH}$, Lindborg SR, et al. How to improve R\&D productivity: the pharmaceutical industry's grand challenge. Nat Rev Drug Discov. 2010;9(3):203-14. https://doi.org/10.1038/nrd3078.

11. Bouvy JC, Koopmanschap MA, Shah RR, Schellekens H. The cost-effectiveness of drug regulation: the example of thorough QT/QTc studies. Clin Pharmacol Ther. 2012;91(2):281-8. https:// doi.org/10.1038/clpt.2011.224.

12. Patel N, Wiśniowska B, Jamei M, Polak S. Real patient and its virtual twin: application of quantitative systems toxicology modelling in the cardiac safety assessment of citalopram. AAPS J. 2017;20(1):6. https://doi.org/10.1208/s12248-017-0155-8.

13. Polak S, Pugsley MK, Stockbridge N, Garnett C, Wisniowska B. Early drug discovery prediction of proarrhythmia potential and its covariates. AAPS J. 2015;17(4):1025-32. https://doi.org/ 10.1208/s12248-015-9773-1.

14. Polak S, Wisniowska B, Fijorek K, Glinka A, Mendyk A. In vitro-in vivo extrapolation of drug-induced proarrhythmia predictions at the population level. Drug Discov Today. 2014;19(3):275-81. https://doi.org/10.1016/j.drudis.2013.10.009.

15. Polak S, Romero K, Berg A, Patel N, Jamei M, Hermann D, et al. Quantitative approach for cardiac risk assessment and interpretation in tuberculosis drug development. J Pharmacokinet Pharmacodyn. 2018;45:457-67. https://doi.org/ 10.1007/s10928-018-9580-2.

16. Polak S, Wisniowska B, Fijorek K, Glinka A, Polak M, Mendyk A. ToxComp+ In vitro-in vivo extrapolation system for drug proarrhythmic potency assessment. In: Computing in Cardiology (CinC). IEEE; 2012. p. 789-92.

17. Malhotra BK, Glue P, Sweeney K, Anziano R, Mancuso J, Wicker P. Thorough QT study with recommended and supratherapeutic doses of tolterodine. Clin Pharmacol Ther. 2007;81(3):377-85. https://doi.org/10.1038/sj.clpt.6100089.

18. Malhotra B, Wood N, Sachse R, Gandelman K. Thorough QT study of the effect of fesoterodine on cardiac repolarization. Int J Clin Pharmacol Ther. 2010;48(5):309-18.

19. Malhotra B, Gandelman K, Sachse R, Wood N, Michel MC. The design and development of fesoterodine as a prodrug of 5hydroxymethyl tolterodine (5-HMT), the active metabolite of tolterodine. Curr Med Chem. 2009;16(33):4481-9.

20. Oishi M, Chiba K, Malhotra B, Suwa T. Effect of the CYP2D6*10 genotype on tolterodine pharmacokinetics. Drug Metab Dispos. 2010;38(9):1456-63. https://doi.org/10.1124/ dmd.110.033407.

21. Mizutani T. PM frequencies of major CYPs in Asians and Caucasians. Drug Metab Rev. 2003;35(2-3):99-106. https:// doi.org/10.1081/DMR-120023681.

22. Brynne N, Dalen P, Alvan G, Bertilsson L, Gabrielsson J. Influence of CYP2D6 polymorphism on the pharmacokinetics and pharmacodynamic of tolterodine. Clin Pharmacol Ther. 1998;63(5):529-39. https://doi.org/10.1016/S0009-9236(98)90104-7.

23. Malhotra B, Dickins M, Alvey C, Jumadilova Z, Li X, Duczynski G, et al. Effects of the moderate CYP3A4 inhibitor, fluconazole, on the pharmacokinetics of fesoterodine in healthy subjects. Br J Clin Pharmacol. 2011;72(2):263-9. https://doi.org/ 10.1111/j.1365-2125.2011.04007.x.

24. ten Tusscher KH, Panfilov AV. Alternans and spiral breakup in a human ventricular tissue model. Am J Physiol Heart Circ Physiol. 2006;291(3):H1088-100. https://doi.org/10.1152/ ajpheart.00109.2006.

25. Polak S, Fijorek K. Inter-individual variability in the pre-clinical drug cardiotoxic safety assessment-analysis of the agecardiomyocytes electric capacitance dependence. J Cardiovasc Transl Res. 2012;5(3):321-32.

26. Polak S, Fijorek K, Glinka A, Wisniowska B, Mendyk A. Virtual population generator for human cardiomyocytes parameters: in silico drug cardiotoxicity assessment. Toxicol Mech Methods. 2012;22(1):31-40.

27. Fijorek K, Patel N, Klima Ł, Stolarz-Skrzypek K, KaweckaJaszcz K, Polak S. Age and gender dependent heart rate circadian model development and performance verification on the proarrhythmic drug case study. Theor Biol Med Model. 2013;10(1):7.

28. Polak S, Fijorek K, Püsküllüoğlu M, Glinka A, Tomaszewska D, Tomaszewski R. Literature review of the serum potassium, sodium and calcium levels in healthy individuals. 2013.

29. Sennels HP, Jørgensen HL, Goetze JP, Fahrenkrug J. Rhythmic 24-hour variations of frequently used clinical biochemical parameters in healthy young males - the Bispebjerg study of diurnal variations. Scand J Clin Lab Invest. 2012;72(4):287-95. https://doi.org/10.3109/00365513.2012.662281.

30. Sweeney KR, Gastonguay MR, Benincosa L, Cronenberger CL, Glue P, Malhotra BK. Exposure-response modeling and clinical trial simulation of the effect of tolterodine on QT intervals in healthy volunteers. Drug Discov Ther. 2010;4(1):44-53.

31. O'Hara T, Virag L, Varro A, Rudy Y. Simulation of the undiseased human cardiac ventricular action potential: model formulation and experimental validation. PLoS Comput Biol. 2011;7(5):e1002061. https://doi.org/10.1371/journal.pcbi.1002061. 